\title{
Sucesión bacteriana del tracto digestivo del lechón alimentado con ensilado biológico
}

\author{
Bacterial succession of the digestive tract of a piglet fed with biological silage \\ Héctor Sánchez Súarez ${ }^{1,3}$, Fredy Fabián Domínguez', Gloria Ochoa Mogollón', \\ Rubén Alfaro Aguilera ${ }^{2}$
}

\section{Resumen}

Se evaluó mediante análisis metagenómico la composición y abundancia de la microbiota bacteriana en tres porciones del tracto gastrointestinal de un lechón, en el alimento balanceado y en ensilados biológicos preparados con residuos de origen acuícola. Se extrajo el ADN de las muestras y se procedió con la secuenciación. La mayor diversidad bacteriana estuvo en el alimento balanceado (44.7\%), predominando Candidatus phytoplasma surgarcane. En el ensilado biológico predominaron las especies Lactobacillus casei y Lactococcus lactis. La diversidad existente fue similar entre el ensilado y el alimento. Se encontró similitudes entre las bacterias de las tres regiones gastrointestinales (L. ultunensis, Selenomonas bovis, Prevotella copri, Enterococcus lactis y Campylobacter sp). En el estómago hubo predominio de Helicobacter rappini y Campylobacter coli, en intestino delgado Selenomonas bovis y Lactobacillus ultunensis y en el recto (heces) predominio de Lactobacillus ultunensis y Prevotella copri.

Palabras clave: metagenómica; ensilado biológico; lactobacilo; sucesión bacteriana

\section{AbSTraCT}

The composition and abundance of the bacterial microbiota in three portions of the gastrointestinal tract of a piglet, in the balanced feed and in biological ensilages prepared with residues of aquaculture origin were evaluated by metagenomic analysis. The DNA of the samples was extracted, and sequenced. The greatest bacterial diversity was in the

\footnotetext{
${ }^{1}$ Departamento Académico de Sanidad Vegetal y Producción Pecuaria, Facultad de Ciencias Agrarias, Universidad Nacional de Tumbes, Perú

${ }^{2}$ Departamento de Biología y Bioquímica, Facultad de Salud, Universidad Nacional de Tumbes, Perú

${ }^{3}$ E-mail: hsanchezs@untumbes.edu.pe
}

Recibido: 11 de abril de 2018

Aceptado para publicación: 10 de octubre de 2018 
balanced feed (44.7\%), with the predominance of Candidatus phytoplasma surgarcane. In the biological silage predominated Lactobacillus casei and Lactococcus lactis. The existing diversity was similar between silage and the concentrate feed. Similarities were found between the bacteria of the three gastrointestinal regions (L. ultunensis, Selenomonas bovis, Prevotella copri, Enterococcus lactis y Campylobacter sp). In the stomach predominated Helicobacter rappini and Campylobacter coli, in the small intestine Selenomonas bovis and Lactobacillus ultunensis and in the rectum (faeces) Lactobacillus ultunensis and Prevotella copri.

Key words: metagenomics; biological silage; lactobacilli; bacterial succession

\section{INTRODUCCIÓN}

El tracto digestivo se coloniza de bacterias a partir del nacimiento del animal, y progresa dependiendo de su ambiente de desarrollo y fuente de alimentación (De Lange $e t$ al., 2010). Posteriormente, estas bacterias se naturalizan, se hacen facultativas y permanentes o se mantienen durante un determinado tiempo de la vida del animal, según la región del tracto intestinal (Fraune y Bosch, 2010; Slifierz et al., 2015). Esta colonización tiene un gran impacto en el estado de salud de los animales, donde ayuda, además, a la maduración del tracto gastrointestinal y cumple un rol importante en el sistema inmunológico (Gardiner et al., 2004; Rosmini et al., 2004; Round y Mazmanian, 2009; Chow et al., 2010; Gebert et al., 2011); generando adicionalmente procesos de exclusión competitiva frente a patógenos $y$, por otro lado, convierten sustancias no digeribles en componentes digestibles que benefician al huésped (Guarner 2007; Ley et al., 2008).

Estudios realizados por Frese et al. (2015), muestran que las comunidades microbianas en lechones lactantes hasta los 21 días de edad son relativamente estables a nivel de familia, siendo más abundantes las poblaciones de Bacteroidaceae, Clostridiaceae, Lachnospiraceae, Lactobacillaceae y Enterobacteriaceae. A partir del día 28, las poblaciones de Bacteroides y Enterobacteriaceae declinan e incrementan las poblaciones de Lactobacillaceae, Ruminococcaceae, Veillonellaceae y Preotellaceae (Koenig et al., 2011). Por otro lado, cuando ocurren casos de cuadros patológicos como diarrea porcina, la diversidad microbiana es afectada, disminuyendo las bacterias correspondientes al Phyla Actinobacteria y Firmicutes, mientras que aquellas del género Enterococcus y bacterias de la familia Enterobacteriaceae son más abundante (HermannBank et al., 2015; Klose et al., 2010).

La metagenómica permite analizar hasta un $99 \%$ la información genética de la gran mayoría de microorganismos que no son cultivables en condiciones de laboratorio (Villamil, 2016). Así mismo, permite estudiar un nicho en particular, conocer qué tipo de bacterias coloniza en determinado ambiente y proporciona información para poder asociar actividades microbianas a ciertas poblaciones (Castillo y Guzmán, 2017)

La presente investigación permitió evaluar, mediante metagenómica, la composición y abundancia de la microbiota bacteriana en varias porciones del tracto gastrointestinal de lechones, asociado con el aporte microbiano del alimento balanceado estándar y la utilización de ensilados biológicos preparados con residuos de origen acuícola. 


\section{Materiales y Métodoos}

De la granja porcina del Centro Pecuario de la Facultad de Ciencias Agrarias de la Universidad Nacional de Tumbes, se seleccionó al azar un lechón cruzado, madre Landrace x Pietrain y padre Yorshire, de 28 días de edad, siete días de haber sido destetado, en buen estado de salud y peso. El animal comenzó a recibir concentrado ad libitum siete días antes del destete. El alimento era preparado en granja utilizando como base maíz, soya y polvillo de arroz (Cuadro 1), cuyo contenido proteico fue de $18 \%$ teórico (15.2\% analizado). Además, se incorporó $15 \%$ adicional de ensilado biológico de residuos del procesamiento de langostino, fermentado con Lactobacillus brevis nativo.

En la obtención del ensilado biológico cocido (E2) se utilizó como materia prima la cabeza de langostino. Para disminuir la carga microbiana se pasó por un hervor hasta cambio de color (5 a $10 \mathrm{~min}$ ), se mezcló según tratamientos $70 \%$ del residuo cocinado (langostino) como sustrato, $25 \%$ de melaza o polvillo de arroz y $5 \%$ de leche con el inoculo bacteriano preparado (Lactobacillus brevis nativo), $0.1 \mathrm{ml} / 1$ leche. La mezcla total se colocó en depósitos plásticos de $200 \mathrm{~L}$ de capacidad, tapándose herméticamente, dejando un $25 \%$ de espacio libre entre el producto y la tapa.

Para el análisis bromatológico se colectó $100 \mathrm{~g}$ del ensilado $\mathrm{E} 2 \mathrm{y}$ de la dieta, y se remitió al Laboratorio de Evaluación Nutricional de Alimentos (LENA) de la Universidad Agraria La Molina (UNALM) (Cuadro 2). Para los fines del estudio, se consideró como ensilado E1 el ensilado biológico crudo.

El lechón fue anestesiado con ketamina, en dosis $3 \mathrm{mg} / \mathrm{kg}$ de peso vivo, vía i.v., para realizar una laparotomía. Se tomaron muestras de mucosa y contenido del estómago, intestino delgado, recto (heces) con hisopos estériles. De igual manera, se colectaron muestras de ensilado biológico y alimento
Cuadro 1. Insumos de dieta utilizada en la alimentación de lechones

\begin{tabular}{lc}
\hline Ingredientes & $\mathrm{kg}$ \\
\hline Grano de maíz & 39.24 \\
Harina de algodón & 3.00 \\
Aceite & 0.50 \\
Polvillo de arroz & 23.71 \\
H. de soja 48\% & 16.50 \\
H. de pescado, AAFCO & 5.00 \\
Concha de ostra & 1.00 \\
Sal común & 0.50 \\
Premezcla vitamínica & 0.05 \\
Premezcla mineral & 0.50 \\
Melaza de caña & 10.00 \\
Ensilado residuo langostino & 15.00 \\
(E2) & 115.0 \\
\hline Total
\end{tabular}

Contenido de proteína $15.22 \%$, según el Laboratorio de Evaluación Nutricional de Alimentos (LENA) de la Universidad Nacional Agraria La Molina (UNALM)

Cuadro 2. Contenido nutricional del ensilado experimental E2

\begin{tabular}{lc}
\hline Nutriente & Contenido \\
\hline Humedad (\%) & 61.12 \\
Proteína (\%) & 42.86 \\
Grasa (\%) & 8.36 \\
Fibra (\%) & 12.42 \\
Cenizas (\%) & 15.05 \\
ELN (\%) & 12.72 \\
\hline
\end{tabular}

Fuente: Laboratorio de Evaluación Nutricional de Alimentos (LENA), Universidad Agraria La Molina (UNALM)

balanceado. Todas las muestras fueron trasladadas en cadena de frío hasta las instalaciones de Inca Biotec S.A.C. (Tumbes), para la extracción del ADN metagenómico.

El ADN metagenómico de las muestras fue extraído con PowerSoil ${ }^{\mathrm{TM}}$ DNA Isolation- 


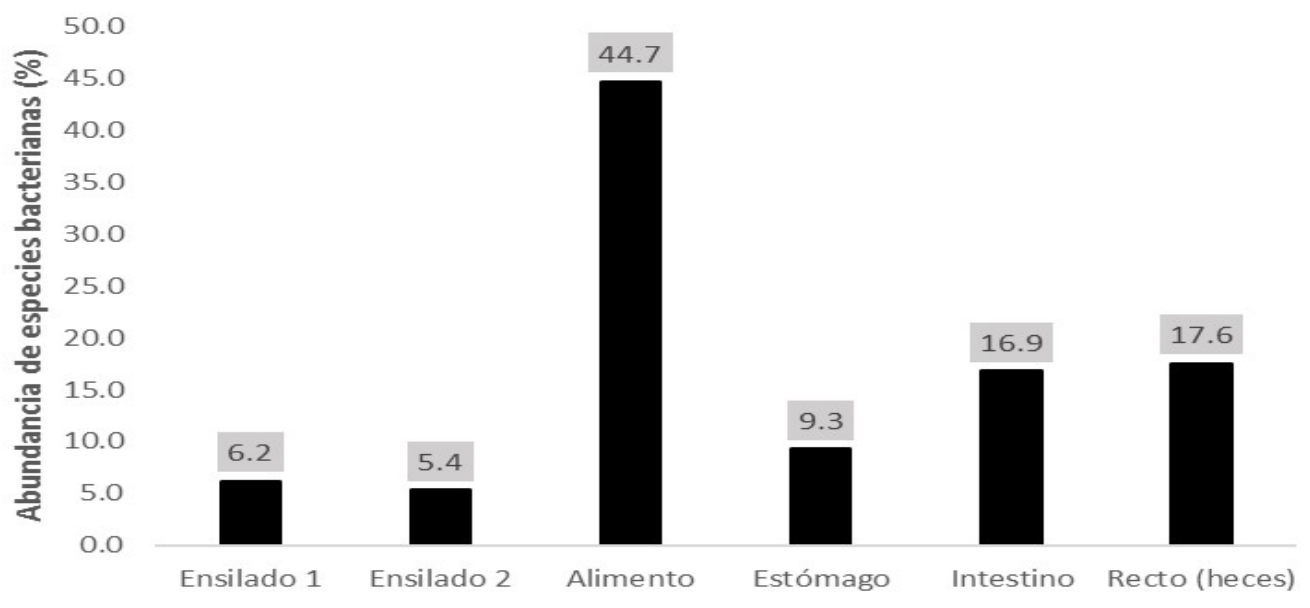

Figura 1. Abundancia de especies bacterianas (\%) en tres matrices biológicas en el lechón (contenido estomacal, intestinal y rectal), en el concentrado, ensilado biológico E1 (residuo crudo) y E2 (residuo precocido).

Kit (MO BIO Laboratories), siguiendo las recomendaciones del fabricante, incluido en el protocolo del laboratorio INCABiotec (Perú). La presencia del ADN extraído fue verificada en geles de agarosa al $1 \%$, teñido con bromuro de etidio $(0.5 \mathrm{~g} / \mathrm{ml})$ y por electroforesis en buffer TAE 1X. Las muestras de ADN fueron remitidas a Mr. DNA Lab. (Molecular Research LP, EEUU), para la secuenciación de metagenomas (Metagenomic Shotgun Sequencing) (16S rRNA Sequencing) mediante la tecnología Diversity Assay bTEFAP ${ }^{\circledR}$ Illumina (Dowd et al., 2008). Los resultados fueron procesados en una hoja Excel, obteniéndose gráficos de porcentaje de abundancia y diversidad.

\section{Resultados}

Se evaluaron seis matrices biológicas, identificándose especies bacterianas cultivables, no cultivables e incluso aquellas que aún no han sido caracterizadas. El mayor número de especies bacterianas identificadas correspondió a la muestra de alimento balanceado ( 685 bacterias, $44.7 \%$ ). Por otro lado, la menor cantidad de cepas identificadas correspondieron a las muestras de ensilados biológicos (E1 y E2) (Figura 1).

La composición de la flora bacteriana en las matrices en estudio fue variable, predominando Candidatus phytoplasma Sugarcane (35\% de abundancia) en el alimento balanceado y Lactobacillus casei y Lactococcus lactis (32 y 42\%, respectivamente) en los ensilados E1 (con residuos crudos) y E2 (con residuos precocidos). Por otro lado, en el tracto digestivo se obtuvo Helicobacter rappini y Campylobacter coli con mayor abundancia en el estómago, Selenomonas bovis y Lactobacillus ultunensis en el intestino delgado y Lactobacillus ultunensis y Prevotella copri en el recto (heces) (Cuadro 2).

En los ensilados biológicos se observó la predominancia de bacterias del género Lactobacillus (L. casei) y Lactococcus ( $L$. lactis). Comparando el ensilado E2 con el alimento balanceado, existe similitud de especies bacterianas, entre ellas L. pontis y Enterococcus faecalis. Por otro lado, aun cuando el ensilado biológico aporta diferentes especies de bacterias del género Lacto- 

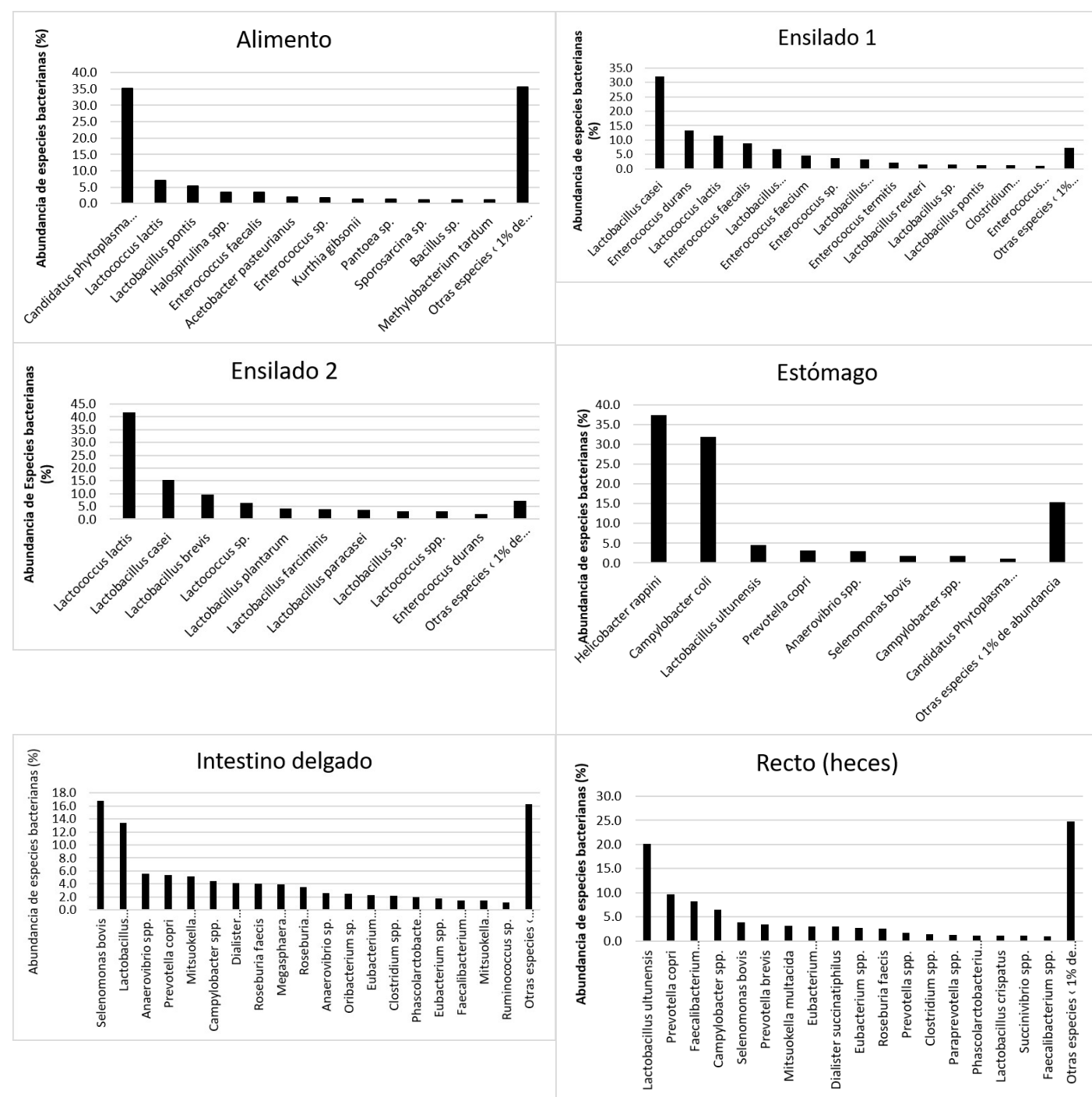

Figura 2. Composición de la flora bacteriana por las matrices biológica de los ensilados E1 (residuo crudo) y E2 (residuo precocido), del alimento balanceado, y de los contenidos estomacal, intestinal y del recto (heces) de un lechón

bacillus, estas no son predominantes en el tracto gastrointestinal del lechón. Así tenemos, que L. ultunensis, además de Selenomonas bovis, Prevotella copri, Enterococcus lactis y Campylobacter spp predominan en estómago, intestino delgado y recto (heces) (Cuadro 3).

\section{Discusión}

Los estudios de la microflora bacteriana en animales de cría se enfocan principalmente en la evaluación de bacterias cultivables en medios de cultivo generales y específicos (Deusch et al., 2015). Sin embargo, actual- 
Cuadro 3. Sucesión de poblaciones bacterianas en las matrices biológica de los ensilados E1 (residuo crudo) y E2 (residuo precocido), del alimento balanceado, y de los contenidos estomacal, intestinal y del recto (heces) de un lechón

\begin{tabular}{|c|c|c|c|c|c|c|}
\hline Población bacteriana & $\begin{array}{c}\text { Ensilado } \\
1\end{array}$ & $\begin{array}{c}\text { Ensilado } \\
2\end{array}$ & Alimento & Estómago & Intestino & Recto \\
\hline Lactobacillus casei & 32.1 & 15.3 & - & - & - & - \\
\hline Enterococcus durans & 13.3 & 2.0 & - & - & - & - \\
\hline Lactococcus lactis & 11.6 & 41.8 & 7.1 & - & - & - \\
\hline Enterococcus faecalis & 8.8 & - & 3.4 & - & - & - \\
\hline Lactobacillus paracasei & 6.9 & 3.6 & - & - & - & - \\
\hline Enterococcus faecium & 4.7 & - & - & - & - & - \\
\hline Enterococcus sp & 3.7 & - & 1.8 & - & - & - \\
\hline Lactobacillus plantarum & 3.2 & 4.2 & - & - & - & - \\
\hline Enterococcus termitis & 2.1 & - & - & - & - & - \\
\hline Lactobacillus reuteri & 1.5 & - & - & - & - & - \\
\hline Lactobacillus sp & 1.4 & 3.1 & - & - & - & - \\
\hline Lactobacillus pontis & 1.3 & - & 5.4 & - & - & - \\
\hline Clostridium perfringens & 1.2 & - & - & - & - & - \\
\hline Enterococcus raffinosus & 1.0 & - & - & - & - & - \\
\hline Lactobacillus brevis & - & 9.6 & - & - & - & - \\
\hline Lactobacillus farciminis & - & 3.8 & - & - & - & - \\
\hline Lactococcus sp & - & 6.4 & - & - & - & - \\
\hline Lactococcus spp & - & 3.0 & - & - & - & - \\
\hline Sporosarcina sp & - & - & 1.2 & - & - & - \\
\hline Pantoea sp & - & - & 1.3 & - & - & - \\
\hline Methylobacterium tardum & - & - & 1.1 & - & - & - \\
\hline Kurthia gibsonii & - & - & 1.4 & - & - & - \\
\hline Halospirulina spp & - & - & 3.5 & - & - & - \\
\hline $\begin{array}{l}\text { Candidatus Phytoplasma } \\
\text { sugarcane }\end{array}$ & - & - & 35.1 & 1.1 & - & - \\
\hline Bacillus sp & - & - & 1.2 & - & - & - \\
\hline Acetobacter pasteurianus & - & - & 2.0 & - & - & - \\
\hline Anaerovibrio spp & - & - & - & 3.0 & 5.5 & - \\
\hline Campylobacter coli & - & - & - & 31.9 & - & - \\
\hline Campylobacter spp & - & - & - & 1.8 & 4.5 & 6.6 \\
\hline Helicobacter rappini & - & - & - & 37.4 & - & - \\
\hline Lactobacillus ultunensis & - & - & - & 4.5 & 13.4 & 20.1 \\
\hline Prevotella copri & - & - & - & 3.2 & 5.4 & 9.7 \\
\hline Selenomonas bovis & - & - & - & 1.8 & 16.8 & 3.9 \\
\hline Mitsuokella multacida & - & - & - & - & 5.1 & 3.2 \\
\hline Dialister succinatiphilus & - & - & - & - & 4.2 & 3.0 \\
\hline Roseburia faecis & - & - & - & - & 4.1 & 2.6 \\
\hline Megasphaera elsdenii & - & - & - & - & 3.9 & - \\
\hline Roseburia intestinalis & - & - & - & - & 3.5 & - \\
\hline Anaerovibrio sp & - & - & - & - & 2.6 & - \\
\hline Oribacterium $\mathrm{sp}$ & - & - & - & - & 2.4 & - \\
\hline Eubacterium ruminantium & - & - & - & - & 2.3 & 3.0 \\
\hline Clostridium spp & - & - & - & - & 2.1 & 1.3 \\
\hline $\begin{array}{l}\text { Phascolarctobacterium } \\
\text { succinatutens }\end{array}$ & - & - & - & - & 2.0 & 1.1 \\
\hline Eubacterium $\mathrm{spp}$ & - & - & - & - & 1.8 & 2.7 \\
\hline Faecalibacterium prausnitzii & - & - & - & - & 1.4 & 8.3 \\
\hline Mitsuokella jalaludinii & - & - & - & - & 1.4 & - \\
\hline Ruminococcus sp & - & - & - & - & 1.1 & - \\
\hline Prevotella brevis & - & - & - & - & - & 3.5 \\
\hline Prevotella spp & - & - & - & - & - & 1.6 \\
\hline Paraprevotella spp. & - & - & - & - & - & 1.3 \\
\hline Lactobacillus crispatus & - & - & - & - & - & 1.1 \\
\hline Succinivibrio $\mathrm{spp}$ & - & - & - & - & - & 1.1 \\
\hline Faecalibacterium spp & - & - & - & - & - & 1.0 \\
\hline $\begin{array}{l}\text { Otras especies }<1 \% \text { de } \\
\text { abundancia }\end{array}$ & 7.3 & 7.2 & 35.6 & 15.4 & 16.2 & 24.8 \\
\hline Total & 100.0 & 100.0 & 100.0 & 100.0 & 100.0 & 100.0 \\
\hline
\end{tabular}


mente existen técnicas moleculares como la metagenómica que permiten conocer la diversidad y estimar la abundancia de microorganismos, tanto cultivables como no cultivables (Escobar-Zepeda et al., 2015). En esta investigación, se describen las especies bacterianas relacionadas con el tracto intestinal del lechón y su relación con las procedentes del alimento balanceado con ensilados biológicos basados en la fermentación generada por bacterias ácido lácticas. Para esto, Kenworthy y Crabb (1963) y Hong (2011) refieren que la colonización bacteriana del aparato gastrointestinal comienza al nacer, cuando el lechón empieza a lactar, y esto está influenciado, además, por el ambiente, la genética de los reproductores y el modo de suministro del alimento balanceado.

Los resultados indican que el alimento balanceado presenta una mayor diversidad de bacterias, comparado con el ensilado y las muestras de contenido intestinal. Esto se debe posiblemente a la naturaleza y calidad de cada uno de los componentes que integran el alimento para animales, que incluye fuente de glúcidos (polvillo, nielen, melaza, maíz), proteínas (torta de soya, pasta de algodón, harina de pescado) y lípidos (aceite de pescado), entre otros aditivos; que aportan microorganismos de diversos orígenes y en diferentes proporciones. Knarreborg et al. (2002) y Lamendela et al. (2011) mencionan que el microbioma intestinal de los animales está relacionado, entre otros, con la dieta y la calidad de los insumos utilizados en su preparación. Así mismo, Scott et al. (2013) indican que la cantidad, tipo y balance de micronutrientes principales de la dieta (glúcidos, proteínas y grasas) tienen un gran impacto en la microbiota intestinal. Otros estudios consideran que el balanceado para animales no es el principal medio que aporta la flora bacteriana al lechón, pues además ocurre a través de la ingesta de las heces de la madre, que dan un gran aporte de microorganismos benéficos y hasta patógenos (Jensen, 1998).
Los ensilados evaluados presentaron una menor diversidad microbiana, debido posiblemente al proceso fermentativo que requiere este producto, el cual es selectivo para algunas especies bacterianas microaerofílicas y anaeróbicas (Ridwan et al., 2015). Dentro de estas, las bacterias de los géneros Lactobacillus (Melgarejo y Velásquez, 2016) y Clostridium presentes en este estudio, cuya predominancia radica en L. lactis y L. casei. Los estudios de Pérez et al. (2007) y Partanen y Mroz (1999) demostraron que estas últimas especies producen metabolitos antimicrobianos que pueden reemplazar a los antibióticos utilizados en la dieta de lechones destetados. Asimismo, L. lactis es utilizada como bacteria recombinante que expresa el factor de crecimiento epidermal porcino (LLpEGF, por sus siglas en inglés) y es utilizado de manera oral en lechones recién destetados, para estimular el desarrollo intestinal, mediante la regulación positiva de la expresión génica de las proteínas de integridad intestinal, las enzimas digestivas y el transporte de nutrientes (Xu et al., 2015).

Aun cuando los preparados de ensilados biológicos se iniciaron a partir de ciertas especies del género Lactobacillus, se presenta el crecimiento de otras especies bacterianas con capacidad probiótica, las que pueden provenir de los insumos utilizados para su preparación. Así se tiene L. ultunensis, que según los resultados obtenidos predomina principalmente en el intestino de los lechones, y no formó parte del inóculo inicial necesario para preparar el ensilado. $L$. ultunensis ha sido aislada a partir de biopsias de mucosa del estómago de humanos sanos (Roos et al., 2005). En forma similar, también ha sido aislada a partir de heces de cerdos y evaluada como posible especie bacteriana con potencial probiótico en esta especie de crianza (de Angelis et al., 2006).

En el tracto gastrointestinal del lechón, específicamente en el estómago, se obtuvo predominancia de Helicobacter rappini. Esta bacteria ha sido aislada principalmente 
en gatos, perros, ratones, ovejas y cerdos (Whary y Fox, 2004). En humanos fue reportado en pacientes con inmunosupresión, generando septicemia bacteriana y enteritis (Tee et al., 2001). Por otro lado, la flora bacteriana del tracto gastrointestinal es predominantemente Gram negativa, que incluye a los géneros Selenomonas y Prevotella como las frecuentemente aisladas a partir del contenido intestinal de cerdos. Según Schaechter (2004), estas bacterias generan un impacto significativo en el metabolismo energético de los cerdos que consumen una dieta rica en granos y fibras, y mejoran la tasa de absorción de ácidos grasos volátiles en el ciego y colon.

\section{Conclusiones}

- Cada uno de los grupos bacterianos identificados es diverso y se encuentran en diferentes proporciones, predominando algunos grupos que permiten mejorar la salud de los cerdos y ayudan al metabolismo de los nutrientes, Por otro lado, algunos de ellos pueden generar impacto negativo en la cría de cerdos con la proliferación de enfermedades de origen bacteriana.

- Se determinó la presencia de microrganismos propios en cada región del tracto digestivo.

- Se encontraron bacterias de las mismas especies en las tres regiones del tracto digestivo, incluyendo algunas con capacidad probiótica.

- Existe una abundante cantidad de lactobacilos en el ensilado biológico de residuos del procesamiento de langostino.

\section{Literatura Citada}

1. Castillo SG, Guzmán G. 2017. Creación de una librería metagenómica del aparato digestivo de Chrysoperla carnea. Jóvenes en la Ciencia 3: 963-968.
2. Chow J, Lee SM, Shen Y, Khosravi A, Mazmanian SK. 2010. Host-bacterial symbiosis in health and disease. Adv Immunol 107: 243-274. doi: 10.1016/ B978-0-12-381300-8.00008-3

3. de Angelis M, Siragusa S, Berloco M, Caputo L, Settanni L, Alfonsi G, Amerio M, et al. 2006. Selection of potential probiotic lactobacilli from pig feces to be used as additives in pelleted feeding. Res Microbiol 157: 792-801. doi: 10.1016/j.resmic.2006.05.003

4. De Lange CFM, Pluske J, Gong J, Nyachoti CM. 2010. Strategic use of feed ingredients and feed additives to stimulate gut health and development in young pigs. Livest Sci 134: 124-134. doi: 10.1016/j.livsci.2010.06.117

5. Deusch S, Tilocca B, Camarinha-Sil$v a A$, Seifert J. 2015. News in livestock research - use of Omics-technologies to study the microbiota in the gastrointestinal tract of farm animals. Comput Struc Biotechnol J 13: 55-63. doi: 10.1016/j.csbj.2014.12.005

6. Dowd SE, Sun Y, Wolcott RD, Domingo A, Carroll JA. 2008. Bacterial tagencoded FLX amplicon pyrosequencing (bTEFAP) for microbiome studies: bacterial diversity in the ileum of newly weaned Salmonella-infected pigs. Foodborne Pathog Dis 5: 459-472. doi: 10.1089/fpd.2008.0107

7. Escobar-Zepeda A, Vera-Ponce A, Sanchez-Flores A. 2015. The road to metagenomics: from microbiology to DNA sequencing technologies and bioinformatics. Front Genetics 6: 348. doi: 10.3389/fgene. 2015.00348

8. Fraune S, Bosch TC. 2010. Why bacteria matter in animal development and evolution. Bioessays 32: 571-580. doi: 10.1002/bies.200900192

9. Frese SA, Parker K, Calvert CC, Mills DA. 2015. Diet shapes the gut microbiome of pigs during nursing and weaning. Microbiome 3:28. doi: 10.1186/ s40168-015-0091-8 
10. Gebert S, Davis E, Rehberger T, Maxwell C. 2011. Lactobacillus brevis strain $1 \mathrm{E} 1$ administered to piglets through milk supplementation prior to weaning maintains intestinal integrity after the weaning event. Benef Microbes 2: 3545. doi: 10.3920/BM2010.0043

11. Gardiner GE, Casey PG, Casey G, Lynch PB, Lawlor PG, Hill C, Fitzgerald GF, et al. 2004. Relative ability of orally administered Lactobacillus murinus to predominate and persist in the porcine gastrointestinal tract. Appl Environ Microb 70: 18951906. doi: 10.1128/AEM.70.4.18951906.2004

12. Guarner F. 2007. Papel de la flora intestinal en la salud y en la enfermedad. Nutr Hosp 22(Suppl 2): 14-19.

13. Hermann-Bank ML, Skovgaard K, Stockmarr A, Strube ML, Larsen N, Kongsted H, Boye M. 2015. Characterization of the bacterial gut microbiota of piglets suffering from new neonatal porcine diarrhoea. BMC Vet Res 11: 139. doi: 10.1186/s12917-0150419-4

14. Hong T, Passoth V, Lindberg J. 2011. Bacterial diversity at different sites of the digestive tract of weaned piglets fed liquid diets. Asian Austral J Anim Sci 24: 834-843. doi: 10.5713/ajas.2011.10291

15. Jensen BB. 1998. The impact of feed additives on the microbial ecology of the gut in young pigs. J Anim Feed Sci 7(Suppl 1): 45-64. doi: 10.22358/jafs/ 69955/1998

16. Kenworthy R, Crabb WE. 1963. The intestinal flora of young pigs, with reference to early weaning, Escherichia coli and scours. J Comp Pathol Therap 73: 215-228. doi: 10.1016/S0368-1742(63)80025-9

17. Klose V, Bayer K, Bruckbeck R, Schatzmayr G, Loibner A. 2010. In vitro antagonistic activities of animal intestinal strains against swine-associated pathogens. Vet Microbiol 144: 515-521. doi: $10.1016 /$ j.vetmic. 2010.02 .025
18. Knarreborg A, Simon MA, Engberg RM, Jensen BB, Tannock GW. 2002. Effects of dietary fat source and subtherapeutic levels of antibiotic on the bacterial community in the ileum of broiler chickens at various ages. Appl Environ Microb 68: 5918-5924. doi: 10.1128/AEM.68.12.5918-5924.2002

19. Koenig JE, Spor A, Scalfone N, Fricker AD, Stombaugh J, Knight R, Angenent LT, et al. 2011. Succession of microbial consortia in the developing infant gut microbiome. P Natl Acad Sci USA 108(Suppl 1): 4578-4585. doi: 10.1073/pnas.1000081107

20. Lamendela R, Domingo JW, Ghosh S, Martinson J, Oerther DB. 2011. Comparative fecal metagenomics unveils unique functional capacity of the swine gut. BMC Microbiol 11: 103. doi: 10.1186/1471-2180-11-103

21. Ley RE, Lozupone CA, Hamady M, Knight R, Gordon JI. 2008. Worlds within worlds: evolution of the vertebrate gut microbiota. Nat Rev Microbiol 6: 776788. doi: $10.1038 \% 2$ Fnrmicro1978

22. Melgarejo Velásquez GY. 2016. Caracterización de bacterias ácido lácticas aisladas del intestino de Litopenaeus vannamei cultivados en sistema intensivo en Tumbes - Perú y su potencial uso como probióticos. Tesis de Biólogo Acuicultor. Nuevo Chimbote, Perú: Univ. Nacional del Santa. 28 p.

23. Partanen KH, Mroz Z. 1999. Organic acid for performance enhancement in pig diets. Nutr Res Rev 12: 117-145. doi: 10.1079/095442299108728884

24. Pérez N, Fajardo P, Méndez J, Cachaldora P, Pastrana L. 2007. Production of four potentially probiotic lactic acid bacteria and their evaluation as feed additives for weaned piglets. Anim Feed Sci Tech 134: 89-107. doi: 10.1016/j.anifeedsci.2006.05.010

25. Ridwan R, Rusmana I, Widyastuti Y, Wiryawan KG, Prasetya B, Sakamoto M, Ohkuma M. 2015. Fermentation characteristics and microbial diversity of 
tropical grass-legumes silages. Asian Austral J Anim 28: 511-518. doi: 10.5713/ ajas. 14.0622

26. Roos S, Engstrand L, Jonsson H. 2005. Lactobacillus gastricus sp. nov., Lactobacillus antri sp. nov., Lactobacillus kalixensis sp. nov. and Lactobacillus ultunensis sp. nov., isolated from human stomach mucosa. Int J Syst Evol Micr 55: 77-82. doi: 10.1099/ijs.0.63083-0

27. Round JL, Mazmanian SK. 2009. The gut microbiota shapes intestinal immune responses during health and disease. Nat Rev Immunol 9: 313-323. doi: 10.1038/ nri2515

28. Rosmini MR, Sequeira GJ, Guerrero I, Martí LE, Dalla R, Frizzo L, Bonazza JC. 2004. Producción de prebióticos para animales de abasto: importancia del uso de la microbiota intestinal indígena. Rev Mex Ing Quim 3: 181-191.

29. Scott K, Gratz S, Sheridan P, Flint H, Duncan S. 2013. The influence of diet on the gut microbiota. Pharmacol Res 69: 52-60. doi: 10.1016/j.phrs.2012.10.020

30. Schaechter M. 2004. The desk enciclopedia of microbiology. China: Elsevier. $1157 \mathrm{p}$.
31. Slifierz MJ, Friendship RM, Weese JS. 2015. Longitudinal study of the earlylife fecal and nasal microbiota of the domestic pig. BMC Microbiol 15: 184. doi: 10.1186/s12866-015-0512-7

32. Tee W, Jenney A, McPhee A, Mijch A, Dyall-Smith M. 2001. «Helicobacter rappini» isolates from 2 homosexual men. Clin Infect Dis 33: e8-e11. doi: 10.1086/320881

33. Villamil C, del Portillo P, Monguí A. 2016. Clonación, expresión y caracterización de una nueva esterasa derivada de metagenomas de suelos agrícolas colombianos. Rev Colomb Biotecnol 18: 48-55. doi: 10.15446/rev.colomb.biote.v18n2.61520

34. Whary M, Fox J. 2004. Natural and experimental Helicobacter infections. Comp Med 54: 128-158.

35. Xu S, Wang D, Zhang P, Lin Y, Fang $Z$, Che L, Wu D. 2015. Oral administration of Lactococcus lactisexpressed recombinant porcine epidermal growth factor stimulates the development and promotes the health of small intestines in early-weaned piglets. J Appl Microbiol 119: 225-235. doi: 10.1111/jam.12833 